\title{
Procedimiento para la medición directa del Rechazo de Modo Común
}

\author{
Procedure for direct measurement of Common Mode Rejection
}

\author{
Enrique Zothner ${ }^{\# 1}$, Julio G. Zola*2, Juan. M. Kelly*3

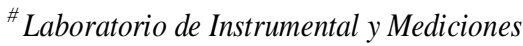 \\ * Laboratorio de Circuitos Electrónicos \\ Universidad de Buenos Aires, Facultad de Ingeniería, Buenos Aires, Paseo Colón 850, Argentina (1063) \\ ${ }^{1}$ ezothn@gmail.com
${ }^{2}$ jzola@fi.uba.ar
${ }^{3}$ jkelly@fi.uba.ar
}

Recibido: 20/09/17; Aceptado: 24/12/17

\begin{abstract}
To measure the Common Mode Rejection (CMR) of an amplifier, it is necessary to carry out the measurements of the implemented and finished circuit, and then perform the measurement of its behavior in real operating conditions. This measurement is necessary to evaluate the susceptibility to conducted electromagnetic interferences (conducted EMI) on the amplifier. In this work, a procedure of direct measurement of the CMR of an amplifier without altering the circuit is described. The measurements are verified by simulation with PSpice.
\end{abstract}

Keywords: CMRR; EMI; Operational Amplifier.

Resumen - Para medir el Rechazo en Modo Común (CMR) de un amplificador, es necesario llevar a cabo las mediciones del circuito implementado y terminado, y entonces realizar la medición de su comportamiento en condiciones de funcionamiento real. Esta medición es necesaria para evaluar la susceptibilidad a las interferencias electromagnéticas conducidas (EMI conducidas) sobre el amplificador. En este trabajo, se describe un procedimiento de medición directa del CMR de un amplificador sin alterar el circuito. Las mediciones se verifican mediante simulación con PSpice.

Palabras clave: CMRR; EMI; Amplificador Operacional.

\section{INTRODUCCIÓN}

Para evaluar parámetros como la susceptibilidad en circuitos expuestos a señales interferentes conducidas (EMI conducidas), es necesario realizar las mediciones en un entorno que se aproxime a la realidad en cuanto a la forma de funcionamiento de dichos circuitos. Uno de los parámetros a evaluar, entre otros, es el rechazo a las señales en modo común, haciéndolo de manera directa sin reconfigurar o alterar el circuito al realizar la medición [1,2].

Sobre estos circuitos deberán evaluarse las amplificaciones para entrada diferencial $\left(A_{d}\right)$ y común $\left(A_{c}\right)$, calculándose el Rechazo al Modo Común $(C M R)$ - también llamado Relación de Rechazo de Modo Común o CMRR -, como se indica en (1):

$$
C M R[d B]=20 . \log \left(A_{d} / A_{c}\right)
$$

Distintas maneras de medición del $C M R$ han sido desde hace tiempo estudiadas en muchas publicaciones. Pero en su mayoría se trabaja en hallar expresiones matemáticas teóricas basadas en mediciones sobre esquemas prestablecidos y no sobre cualquier esquema circuital $[3,4,5,6,7,8]$.

El conjunto de prueba propuesto en este trabajo se basa en aislar los instrumentos de medición (y el circuito mismo) de la red de alimentación de alterna mediante transformadores.

El uso de transformadores de aislación en el banco de medición, permite utilizar el generador de excitación de entrada, tanto en forma diferencial como en modo común.

Se implementa el mismo esquema para conocer el comportamiento del amplificador con señales interferentes dentro y fuera de la banda de señal útil.

\section{PRINCIPIOS GENERALeS}

El valor del $C M R$ de un amplificador operacional (OPAMP), medido por el fabricante en condiciones de lazo abierto, no puede utilizarse directamente para conocer el $C M R$ de circuitos amplificadores que lo incluyen, sean estos con entrada balanceada (amplificador diferencial) o desbalanceada. Esto se debe a distintas causas: 
- Las corrientes de modo común se cierran a tierra a través de las capacitancias parásitas existentes en el circuito. Por lo que la influencia del modo común será dependiente de la distribución de componentes (layout) [9].

- En amplificadores de entrada balanceada, hay que agregar el hecho de que la dispersión en los valores de los componentes (por ejemplo, los resistores de un amplificador de instrumentación) tienen una fuerte influencia en el comportamiento de las señales de modo común [10].

Entonces, el CMR del circuito podría verse fuertemente degradado respecto de sus condiciones ideales, por lo que resultaría necesario medir el valor real del $C M R$.

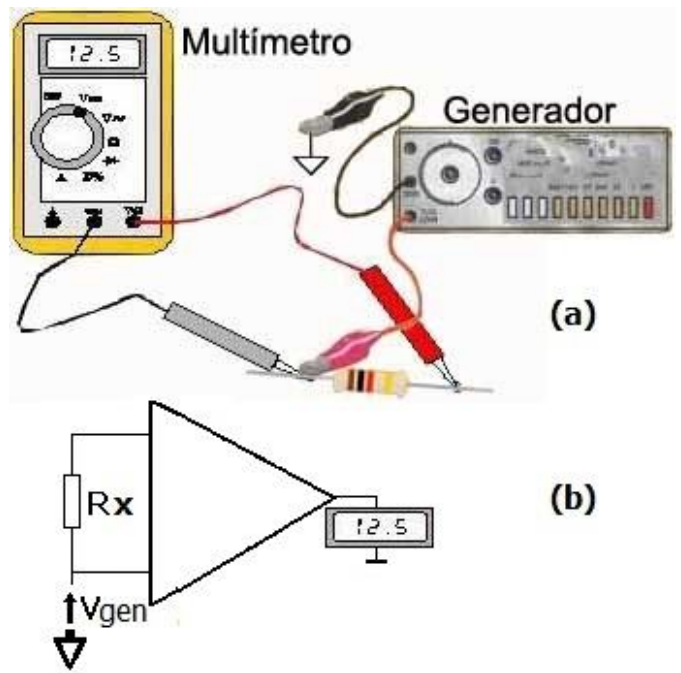

Fig. 1. Evaluación de $C M R$ en la medición de resistencia con multímetro. (a) Banco de medición. (b) Esquema circuital.

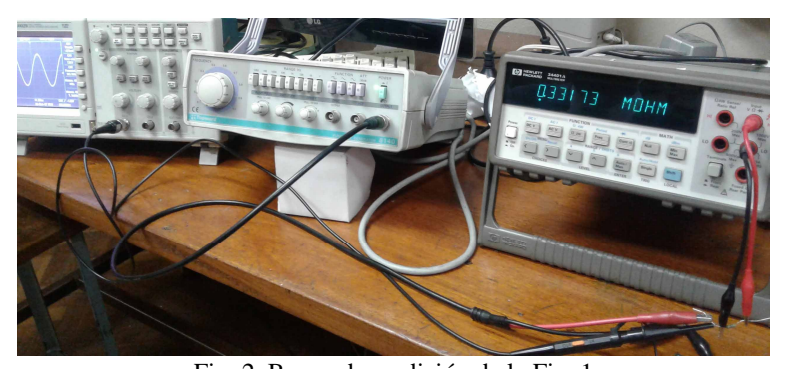

Fig. 2. Banco de medición de la Fig. 1

En la Fig. 1 se muestra un esquema básico para ejemplificar la influencia del modo común en la medición del valor de resistencia medido con un multímetro. El multímetro, aislado de tierra, mide el valor de la resistencia a través de su amplificador diferencial. Si se le agrega la señal de un generador a uno de los terminales, respecto de tierra, el valor medido podría verse modificado, lo que pondría en evidencia la influencia del $C M R$ del multímetro. La Fig, 2 muestra una imagen de este banco de medición, donde se agregó un osciloscopio para monitorear la señal del generador.
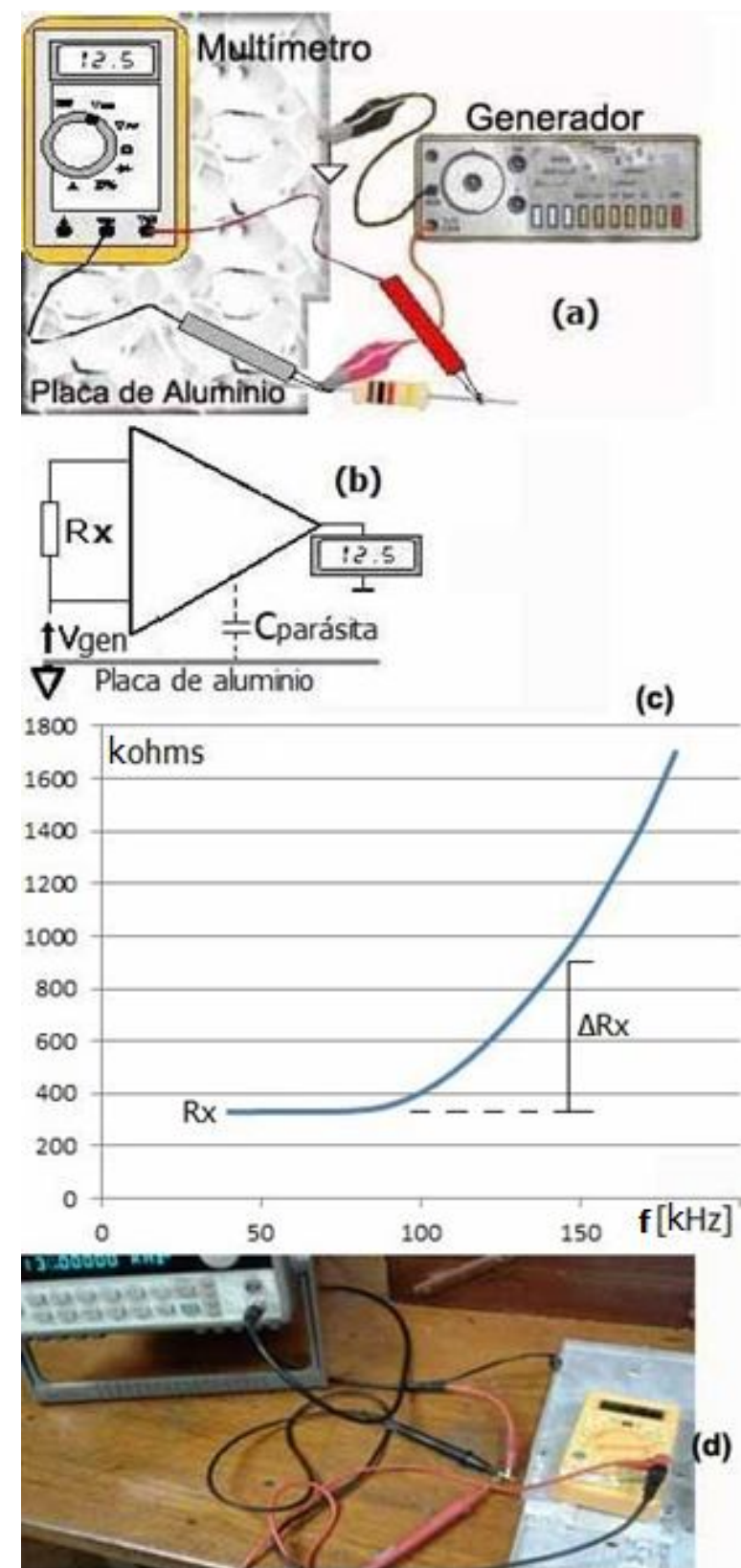

Fig. 3. Evaluación de $C M R$ en la medición de resistencia con multímetro sobre una placa de aluminio conectada a tierra. (a) Banco de medición. (b) Esquema circuital. (c) Valor medido. (d) Imagen del banco de medición.

En esta medición de ejemplo se utilizó un multímetro HP de ancho de banda $1 \mathrm{MHz}$, para medir un resistor de $330 \mathrm{k} \Omega$. La medición en todo el ancho de banda fue de $332,58 \mathrm{k} \Omega$, pero al agregar una señal de 8 Vrms y frecuencia máxima 
de la banda desde un generador de funciones, el valor indicado por el multímetro se modificó a $331,78 \mathrm{k} \Omega$. Es decir, hubo un corrimiento en el valor medido del $0,24 \%$.

En la Fig. 3a se muestra un esquema de un segundo ejemplo, donde se realizó nuevamente la medición del resistor de $330 \mathrm{k} \Omega$, montando el multímetro sobre una placa de aluminio puesta a tierra a través del generador de funciones. De esta forma se intenta poner en mayor evidencia el efecto de capacitancias parásitas que influyen en el valor del $C M R$ (Fig. 3b).

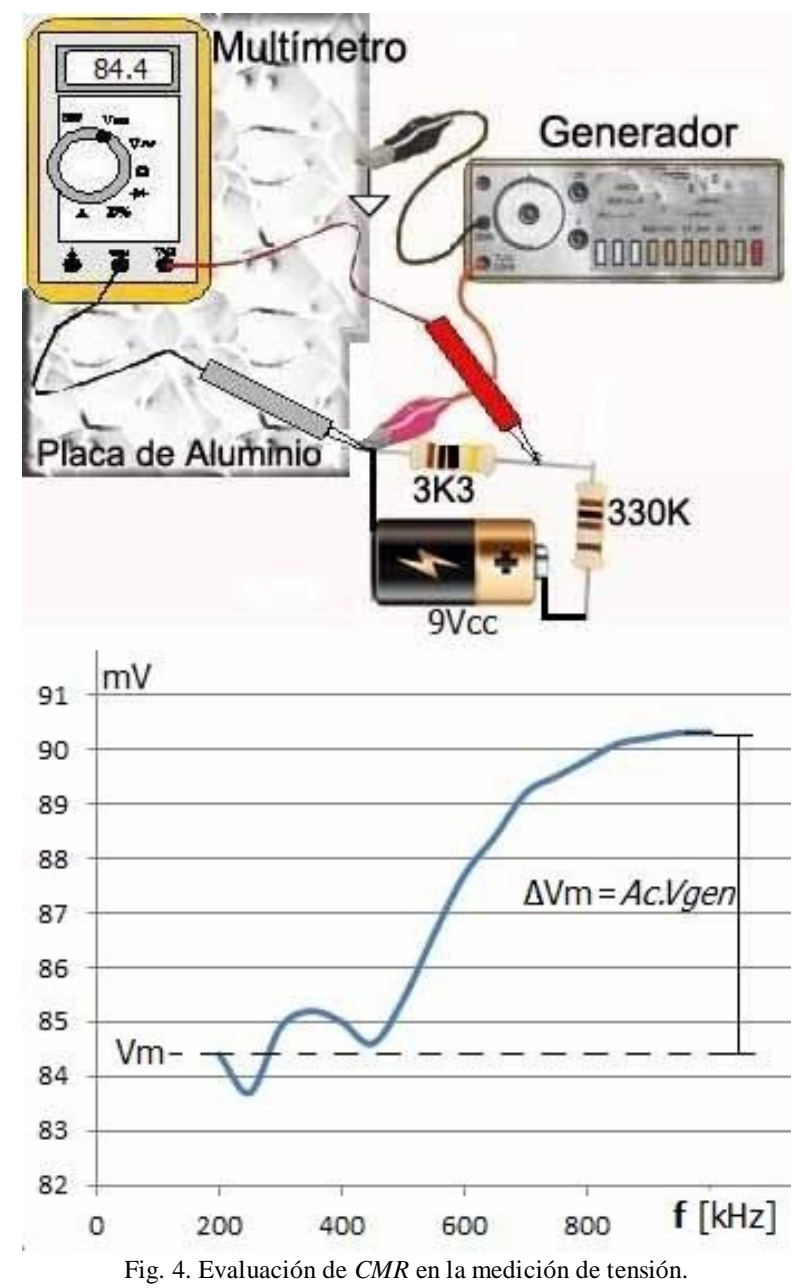

En este caso se utilizó un multímetro UNI-T de bajo costo, aplicándose una tensión senoidal interferente de modo común de 3,5Vrms a través del generador para distintos valores de frecuencia, obteniéndose una gráfica del valor medido como la indicada en la Fig.3c. La Fig, 3d muestra la imagen del banco de medición. Cabe aclarar que la misma medición realizada para valores de señal senoidal interferente de menor valor, arrojó una variación menor del valor medido y con $2 \mathrm{Vrms}$, el valor medido permaneció casi inalterado. Del mismo modo, la desconexión a tierra de la placa de aluminio hace que la variación del valor medido sea mucho menor a la que se muestra en la gráfica.

Por último, la Fig, 4 muestra el esquema de un tercer ejemplo de medición de una tensión continua de unos $85 \mathrm{mVcc}$, realizada con el mismo multímetro y aplicando una señal de modo común de 3,5Vrms para distintos valores de frecuencia. Tal como se observa en la gráfica de la figura, la diferencia entre el valor medido en bajas frecuencias (o en ausencia de señal de modo común) y el valor indicado por el multímetro a frecuencias más elevadas, estaría asociado al CMR del multímetro.

En síntesis, estos ejemplos muestran que las condiciones de funcionamiento pueden alterar la respuesta de un amplificador a las señales de modo común. Asimismo, de acuerdo a la medición, podría expresarse la $C M R$ como proporcional a $R x / \Delta R x$ o $V m / \Delta V m$, según el multímetro se lo utilice como óhmetro o voltímetro, respectivamente.

Cabe aclarar que, si bien el ejemplo de la medición de resistencia pone en evidencia el problema, el agregado del ejemplo de medición de tensión puede resultar de más simple interpretación puesto que no entran en juego los circuitos anexos del óhmetro. Por otro lado, las imágenes con el instrumental utilizado son al solo efecto de mostrar que es un esquema simple de medición con instrumental replicable en cualquier laboratorio.

\section{ANÁLISIS TEÓRICO}

Tal como se mencionó anteriormente, los métodos para la medición del CMR descriptos en la bibliografía, se basan en la reproducción de la configuración teórica de un amplificador diferencial, donde se analiza el valor del voltaje de salida ante señales de entrada en modo diferencial y común [5].

Cuando el circuito amplificador deja de tener una entrada balanceada, la reproducción de señales de modo diferencial y común no es tan simple de realizar sin modificar el circuito, lo cual podría cambiar su comportamiento respecto a la configuración real de funcionamiento.

Otra forma de obtención del CMR que también se utiliza en circuitos amplificadores no 
balanceados, es la de medir la amplificación que se admite aproximadamente igual a $A_{d}$ y obtener $A_{c}$ mediante el valor del error entre el valor medido de $A_{d}$ y el valor teórico calculado. Este método dependerá del tipo de instrumental empleado y del error con que se realicen las mediciones [3].

El procedimiento propuesto en este trabajo, se basa en considerar que, en funcionamiento real, el amplificador se verá afectado por señales de modo común respecto de tierra en todos sus terminales, no solamente en la entrada, debido a corrientes que circulan a través de las capacitancias parásitas equivalentes entre los terminales del circuito y tierra (EMI conducidas).

Por ejemplo, en la Fig. 5a se muestra un amplificador no inversor, donde se puede ver la acción de las señales de modo común, conjuntamente con la señal útil $V i$.

En la Fig. 5b se muestra un esquema simplificado para analizar la influencia del modo común. En esta figura, se puede observar que para el modo común, da lo mismo si el amplificador es inversor o no inversor, lo que permite un análisis más general, independiente de la configuración.

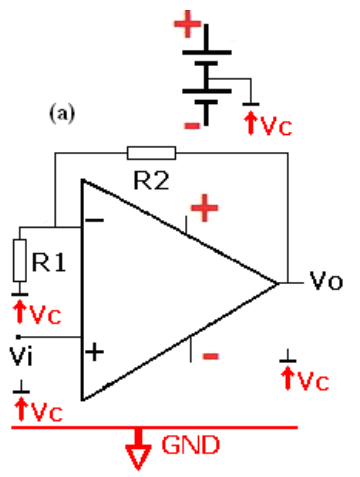

(b)

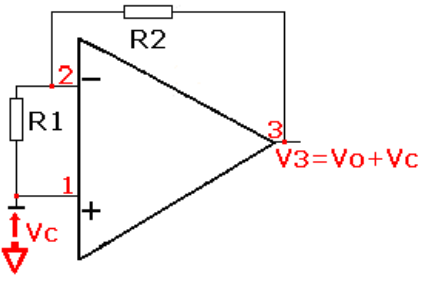

Fig. 5. (a) Modo común sobre un circuito real. (b) Esquema simplificado.

Cabe aclarar que se hace foco en el análisis de $A_{c}$, ya que la amplificación de la señal útil del circuito, $V o / V i$, será prácticamente $A_{d}$, la cual es simple de obtener en una medición directa. En el caso de la Fig. 5b, se puede expresar Vo como (2):

$$
v_{3}-v_{c}=v_{o} \approx v_{c} A_{c}
$$

La expresión (2) indica que para medir directamente $A_{c}$, se debería conectar un instrumento aislado de tierra entre la salida y el terminal común del circuito, como por ejemplo un voltímetro, como se indica en la Fig. 6. Cabe aclarar que algunos multímetros tienen implementado la medición de verdadero valor eficaz, otros son de valor medio y están calibrados a valor eficaz. Es muy importante conocer de qué tipo es el multímetro, especialmente si se está midiendo señales con ruido, como puede ser este caso. Por ello el multímetro utilizado mide verdadero valor eficaz.

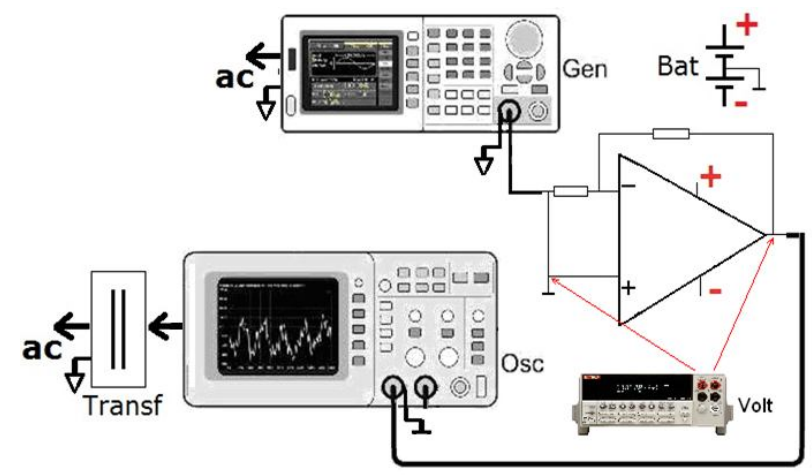

Fig. 6. Esquema genérico de medición.

Resulta importante el hecho de aislar dicho instrumento conectado a la red de alimentación de alterna mediante un transformador de aislación con muy baja capacitancia parásita entre primario y secundario, ya que en caso de una conexión directa agregaría un nuevo componente de modo común: corrientes de $50 \mathrm{~Hz}$ y sus armónicos que circularían por el instrumento y el circuito a través de tierra.

Aun así, las corrientes de $50 \mathrm{~Hz}$ podrían estar presentes circulando a través de la alimentación de corriente continua del amplificador, aspecto que debe ser tenido en cuenta en el diseño de dicho circuito. En este trabajo, se alimentó al amplificador con baterías.

Nótese que las simbologías para el común y para la tierra difieren. Es muy importante recordar que la de forma triangular es tierra y puede estar referida a una jabalina, mientras que la que se representa con una rayita horizontal es la referencia común de las tensiones del circuito que en este caso está aislada de tierra por el transformador de aislación.

En el ejemplo de la Fig. 6, el osciloscopio aislado de la red de alimentación eléctrica mediante un transformador se lo muestra como un auxiliar para monitorear la forma de la señal de salida respecto del terminal común. Se utilizó este tipo de instrumento ya que permite visualizar algún malfuncionamiento del circuito bajo prueba, 
como ruido, oscilaciones, saturación, deformación de la onda, etc., que con el multímetro quedaría enmascarado.

Puede observarse, de acuerdo con este análisis teórico, que la limitación en cuanto al valor medido de $A_{c}$ estaría dado por el $C M R$ propio del voltímetro utilizado.

\section{MEDICIONES Y RESULTADOS}

Dado que se presupone un $C M R$ elevado para el amplificador (teniendo en cuenta que se conoce que para el OPAMP utilizado se especifica un valor superior a $\operatorname{los} 80 \mathrm{~dB}$ en el ancho de banda útil), la señal necesaria para la excitación en modo común tiene que ser de un valor lo más alto posible que permita una respuesta del amplificador aceptable para ser medida. En este caso se utilizó para las pruebas una señal entre 2 y 8 Vrms respecto del terminal de tierra, es decir entre 5,5 y $22 \mathrm{Vpp}$, estando el amplificador alimentado con baterías entre $\pm 9 \mathrm{~V}$ respecto del terminal de referencia común.

Entonces, mediante el esquema de medición de la Fig. 6 y utilizando un generador senoidal de frecuencia variable, un osciloscopio aislado de la red eléctrica y un multímetro digital de 6 1/2 dígitos y que tiene $1 \mathrm{MHz}$ de ancho de banda, se procedió a medir la tensión de salida del amplificador respecto del terminal común.

Se calcularon los valores de $A_{c}$ en función de la frecuencia como están indicados en la Fig. 7a (curva azul). Se utilizaron para ello OPAMP de la serie TL082 y OPA2277.

En la misma gráfica se muestran además las curvas en rojo y verde correspondientes a los resultados de la simulación en PSpice [12] del circuito bajo prueba. En la simulación se consideraron capacitancias parásitas $C p$ entre cada terminal y tierra, tal como se indica en el esquema circuital de la Fig. $7 \mathrm{~b}$, de $100 \mathrm{pF}$ y $500 \mathrm{pF}$ respectivamente. Si bien se consideraron para simplificar todas las capacitancias de igual valor, la más influyente resultó ser $C p l$ y puede observarse que el aumento de esta capacidad aproxima la curva simulada de $A_{c}$ al valor medido.

La Fig. 7a resulta similar para el TL082 y el OPA2277 evaluados.

Por otra parte, el valor de $A_{d}$ puede medirse fácilmente conectando la señal de entrada $V i$ como se indica en la Fig. 5a o bien calcularse mediante la relación entre $R_{1}$ y $R_{2}$. Para $R_{1} \cong 1 \mathrm{k} \Omega$ y $\mathrm{R}_{2} \cong 10 \mathrm{k} \Omega$ la medición de $A_{d} \cong 11$ resultó constante para todo el rango de frecuencias barrido.

De este modo, la gráfica de $A_{c}$ brinda información directa de la variación del $C M R$. Es decir, $C M R$ disminuye con la frecuencia a medida que aumenta $A_{c}$, calculándose un valor máximo de $C M R \cong 57 \mathrm{~dB}$.

Estos valores de $A_{c}$ confirman que el elevado valor de $C M R$ de los OPAMP indicado en sus especificaciones queda enmascarado por los elementos constitutivos del circuito completo incluyendo las capacidades parásitas que se ven reflejadas en la respuesta de $A_{c}$ con la frecuencia.

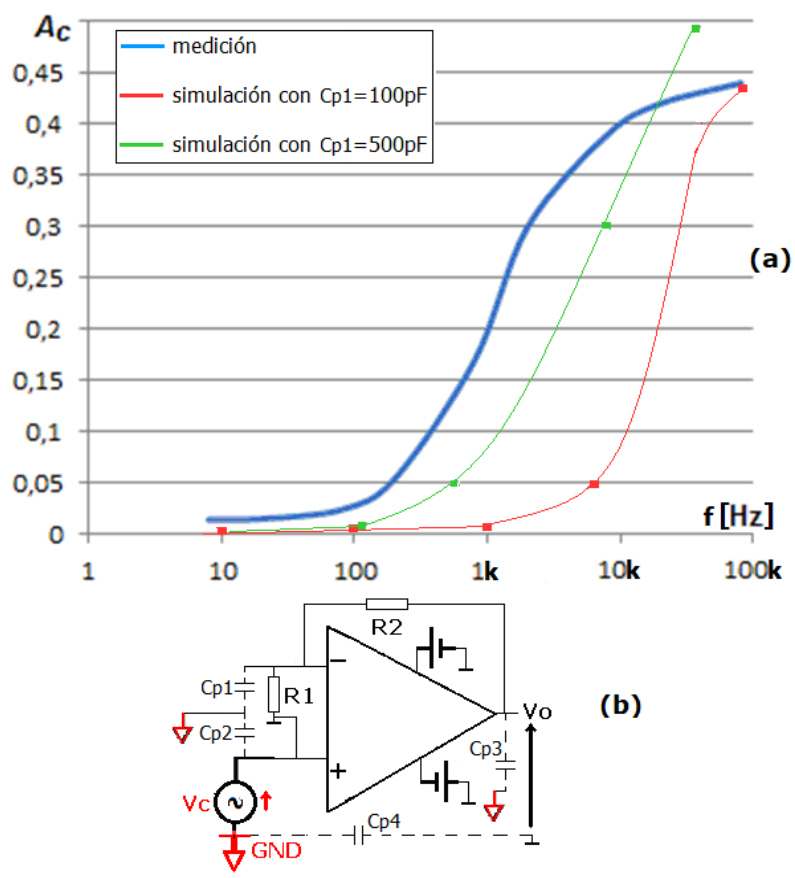

Fig. 7. (a) Medición de $A_{c}$ de un OPAMP TL082 con entrada no balanceada (curva azul) y simulaciones para dos valores de capacitancia parásita $C p 1$. (b) Circuito de simulación agregando $C p$.

En el caso de un amplificador con entrada balanceada, puede realizarse un procedimiento directo similar, modificando la señal de entrada en diferencial y común, mediante el uso de llaves de conexión, como se indica en la Fig. 8.

En la Fig. 8a se muestra una típica configuración de un amplificador de instrumentación diferencial. En este caso se utilizó una relación de 10 entre $R_{2} / R_{1} \cong R_{4} / R_{3} \cong$ 10 a los fines de comprobar los valores medidos en base a una configuración conocida del amplificador y así verificar el método de medición propuesto. Para realizar el cálculo y verificar el funcionamiento, teniendo en cuenta que la diferencia entre los valores de las 
resistencias establece el $C M R$ en bajas frecuencias, se introdujo una diferencia conocida de forma de calcular teóricamente el $C M R$ y luego verificar con el circuito propuesto los valores medidos.

Para verificar el funcionamiento del conjunto de prueba se utilizó esta configuración típica, donde se midieron previamente cada uno de los resistores componentes que se utilizaron, ya que, como se aclaró previamente, la disparidad respecto a sus valores nominales (entre $\pm 1 \%$ o $\pm 5 \%$, por ejemplo) influye directamente en el valor del $C M R$ del conjunto OPAMP más resistores [10], resultando (3), expresión dependiente de la disparidad entre los resistores [11]:

$$
C M R \approx \frac{R_{1} R_{4}+R_{2} R_{3}+2 R_{2} R_{4}}{2\left(R_{1} R_{4}-R_{2} R_{3}\right)}
$$
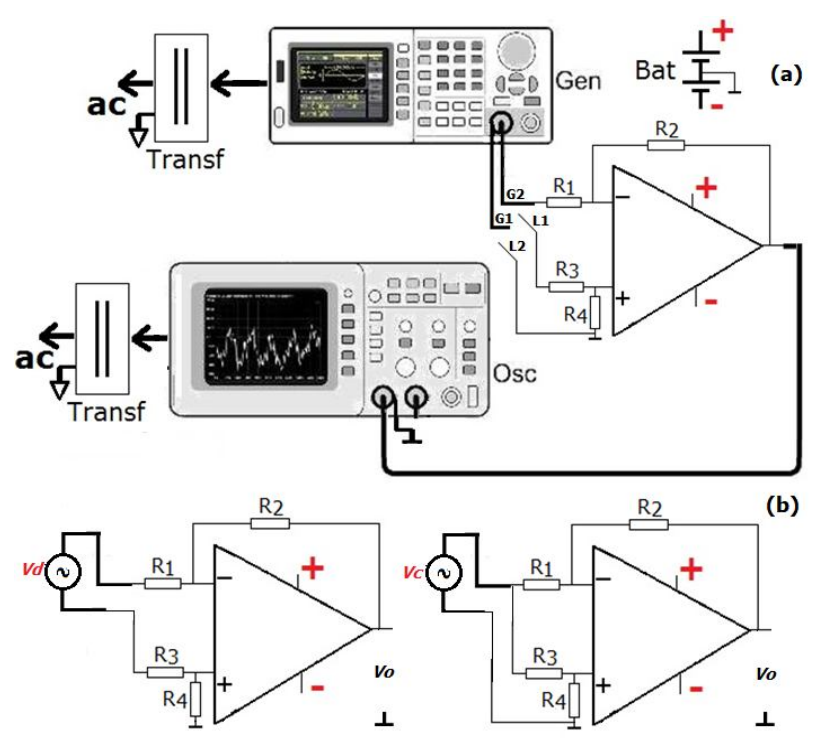

Fig. 8. (a) Esquema de medición para un amplificador diferencial. (b) Conexión de las llaves para modo diferencial y común.

Esto hace que el valor elevado del $C M R$ del OPAMP quede enmascarado frente a la diferencia en el valor de los resistores (sin considerar aún efectos de capacitancias parásitas).

Para realizar la medición de este circuito con entrada balanceada se utilizó para excitar el amplificador bajo prueba, un generador senoidal de frecuencia variable y para la medición de la respuesta, un osciloscopio conectado a través de un transformador de aislación, como se indica en la Fig 8a.

Nótese nuevamente que la referencia del osciloscopio no está conectada a tierra sino a un potencial que no tiene conexión galvánica con la tierra. Esto permite realizar la medición propuesta, aunque el transformador de aislación introduce como efecto indeseado una capacidad parásita entre la tierra y la referencia.

De acuerdo con el esquema de llaves, el generador puede ser utilizado tanto para excitación diferencial como para la común siendo los esquemas equivalentes los mostrados en la Fig. 8b. Para entrada diferencial, L1 se conecta al terminal G1, permaneciendo L2 abierta. Para entrada común, L1 se conecta a G2 y L2 a G1.

Se optó por alimentar al amplificador por baterías de $9 \mathrm{~V}$ en lugar de utilizar fuentes de alimentación conectadas a la red mediante un transformador de aislación. Esto facilita la medición y reduce los errores que introducen las capacidades parásitas de los transformadores de aislación.

Por otro lado, los valores de las señales de excitación entregadas por el generador aislado se eligieron de acuerdo con la ganancia del amplificador y la máxima señal del modo diferencial y común admisibles. En este caso, para la obtención de $A_{c}$ se aplicó una señal de entrada del generador de excitación respecto de común de varios Volts (dentro del rango de modo común del OPAMP), y en el caso de $A_{d}$, por su valor elevado, fue suficiente una señal de cientos de $\mathrm{mV}$.

Este esquema permite utilizar el canal del osciloscopio hasta su sensibilidad máxima. En la medición realizada a pesar de medirse señales de poca amplitud, la señal útil no llegó a ser enmascarada por la señal de $50 \mathrm{~Hz}$ de la red de alimentación o sus componentes armónicos.

Con el esquema de la Fig. 8a se midió $A_{d}$ y luego $A_{c}$ y se varió la frecuencia de excitación para conocer el $C M R$ a distintas frecuencias.

TABLA I

VALORES MEDIDOS USANDO EL ESQUEMA DE LA FIG. 8 PARA $\mathrm{R}_{1} \cong 985 \Omega, \mathrm{R}_{2} \cong 9,85 \mathrm{~K} \Omega, \mathrm{R}_{3} \cong 981 \Omega \mathrm{Y} \mathrm{R}_{4} \cong 9,87 \mathrm{~K} \Omega$

\begin{tabular}{|l|l|l|l|l|l|l|l|}
\hline$f[\mathrm{~Hz}]$ & 0,5 & 5 & 50 & 500 & $5 \mathrm{k}$ & $50 \mathrm{k}$ & $500 \mathrm{k}$ \\
\hline$A_{d}$ & 10 & 10 & 10 & 10 & 10 & 10 & 7,5 \\
\hline$A_{c}\left[10^{-3}\right]$ & 4,8 & 5,1 & 5,8 & 5,8 & 5,9 & 7,7 & 21 \\
\hline$C M R[\mathrm{~dB}]$ & 66,4 & 65,8 & 64,7 & 64,7 & 64,6 & 62,3 & 50,7 \\
\hline
\end{tabular}

En la Tabla I se muestran los resultados de los valores medidos para distintas frecuencias de $A_{d}$, $A_{c}$ y el valor del $C M R$ correspondiente. Tal como se indica en la Tabla I, los valores medidos se obtuvieron utilizando los siguientes valores de 
resistencia: $R_{1} \cong 985 \Omega, R_{2} \cong 9,85 \mathrm{k} \Omega, R_{3} \cong 981 \Omega$ y $\mathrm{R}_{4} \cong 9,87 \mathrm{k} \Omega$.

En la Fig. 9 se muestran los resultados de la simulación en PSpice [12] (línea azul) del amplificador bajo prueba $\left(A_{d}\right.$ y $A_{c}$ en función de la frecuencia), según los circuitos mostrados en la Fig. 8b, agregando en cada caso las capacitancias parásitas $C p$ entre cada terminal y tierra, tal como el caso de la Fig. 7b (se omitió representarlas en la Fig. 8b para una más sencilla interpretación del esquema circuital).

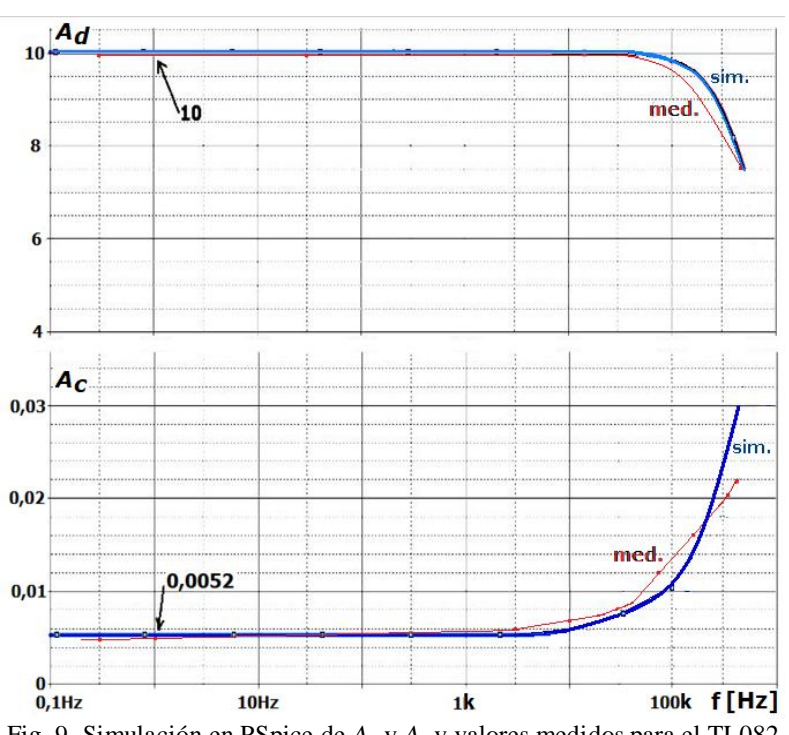

Fig. 9. Simulación en PSpice de $A_{d}$ y $A_{c}$ y valores medidos para el TL082

En línea roja se grafican los valores de la Tabla 1. Puede notarse que los valores de $C M R$ calculados a partir de la simulación $(65,7 \mathrm{~dB}$ en bajas frecuencias y $48 \mathrm{~dB}$ a $500 \mathrm{kHz}$ ), resultan similares a los obtenidos en la medición. Las simulaciones realizadas con el modelo del OPAMP TL082 y con el OPA2277 resultaron similares (en la figura se muestra los resultados para el TL082). El resultado de la simulación que mejor se aproximó a la medición de $A_{c}$ resultó para un desbalance entre las capacitancias parásitas de entrada $C p 1$ y $C p 1$ de algunos pocos $\mathrm{pF}$. Este resultado es esperable ya que estas capacidades de entrada desbalancean las impedancias del amplificador balanceado. Dicho desbalance no alteró prácticamente la respuesta en la simulación de $A_{d}$.

\section{Conclusiones}

La medición del Rechazo de Modo Común, para evaluar la susceptibilidad de un amplificador ante señales interferentes, resulta en general en procedimientos que modifican la topología del circuito. Por lo tanto, el valor resultante de la medición podría no ser el mismo a aquel cuando el amplificador se encuentra en funcionamiento real, en especial en los casos en que la entrada no es balanceada. El procedimiento propuesto permite la medición directa de la amplificación de modo común como una EMI conducida aplicada al circuito. Dicho procedimiento resulta aplicable a amplificadores con entrada balanceada o no balanceada, indistintamente.

Asimismo, conviene resaltar que este trabajo solamente se ha utilizado la herramienta de simulación para la comprensión de los resultados de los valores medidos del circuito bajo prueba. No se está analizando sólo la respuesta de un amplificador operacional sino la del circuito donde éste es una parte de los componentes que hacen al amplificador.

En el modelo de simulación se tuvieron en cuenta los efectos parásitos de capacitancias del circuito bajo prueba. La medición del CMR tal como se propone en distintos papers y notas de aplicación solo brinda información del $C M R$ del amplificador operacional, mientras que en este trabajo se propuso medir el $C M R$ del circuito donde el amplificador operacional es una parte componente.

Tal como se dijo, para obtener los resultados indicados en las simulaciones, especialmente en la obtención de la amplificación de modo común, se agregaron valores de capacitancias parásitas entre los distintos terminales del circuito y tierra que pusieron en evidencia su influencia en las gráficas. Sin estos elementos parásitos, el resultado de la simulación para $A_{c}$ difiere notablemente de la gráfica obtenida mediante medición. Es decir, los resultados de la simulación se aproximan a los valores medidos al agregar al circuito las capacidades parásitas. Tampoco es el objetivo de este trabajo hallar los valores exactos de las capacitancias parásitas, que variarían con la distribución de componentes e incluso podría esperarse con la conexión del instrumental en particular, pero sí mostrar su influencia sobre el parámetro medido.

\section{REFERENCIAS}

[1] R. Pallas, J. Webster, "Common mode rejection ratio in differential amplifiers", IEEE Transactions on Instrumentation and Measurement, Vol. 40, No. 4, Aug 1991.

[2] P. Gray, P. Hurst, S. Lewis, R. Mayer, "Analysis and Design of Analog Integrated Circuits", 5th Ed., Wiley \& Sons., 2009. 
Revista elektron, Vol. 2, No. 1, pp. 39-46 (2018)

[3] Analog Devices, "Op Amp Common-Mode Rejection Ratio (CMRR)", MT-042 Tutorial, 2000.

[4] B. Baker, "Measuring amplifier DC offset voltage, PSRR, CMRR, and open-loop gain", EDN Network, 2014.

[5] J. Zhou, J. Liu, "On a measurement of Common-Mode Rejection Ratio”, IEEE CAS, Vol. 52, No. 1, January 2015.

[6] Texas Instruments, "What you need to know about CMRR - the operational amplifier", TI E2E Community, 2013.

[7] B. Baker, "Understanding CMR and instrumentation amplifiers", EDN Network, 2009.
[8] A. Saab, "Experiments suggest methods for CMRR measurement", EE Times, 2004.

[9] Pulse Technitrol Company, "Understanding Common Mode Noise", G019, April 1999.

[10] E. Nash, "A Practical Review for Common Mode and Instrumentation Amplifiers", www.sensormags.com, July 1998.

[11] K. Lokere, T. Hutchison, G. Zimmer, "Precision Matched Resistors Automatically Improve Differential Amplifier CMRR - Here's How", Linear Technology Design Note 1023., 2013.

[12] OrCAD.com, "OrCAD-PSpice Designer Lite (OrCAD \& PSpice only)", 2016. 